\title{
EFECTO DEL TIPO DE AGENTE DE PELADO, TIEMPO DE COCCIÓN Y CONTENIDO DE HUMEDAD EN LA CALIDAD DE SNACKS FRITOS DE MAÍZ BLANCO GIGANTE (Zea mays)
}

\author{
Geimy I. Boluarte Alarcón, Cecilia P. Dionisio Fernández, y Fausto H. Cisneros*
}

\begin{abstract}
RESUMEN
Con el objetivo de mejorar las características organolépticas del snack frito de maíz gigante, se estudiaron los efectos del tipo de agente de pelado químico $(\mathrm{CaOH}$ o $\mathrm{NaOH})$, el remojo en la solución de pelado, el tiempo de cocción y contenido de humedad del grano antes de la fritura.

Se encontró que $\mathrm{NaOH}$ era más eficiente que $\mathrm{CaOH}$ para el pelado químico del maíz, y cuando se dejaban remojando los granos por $6 \mathrm{~h}$ en la solución de pelado de $\mathrm{NaOH}$, se desarrollaba mejor el sabor nixtamalizado, y además se obtenía un color más claro del snack. Un mayor tiempo de cocción $(0,8,16$ min), producía una mayor expansión del snack, y el ajuste del contenido de humedad mediante el secado, previo a la fritura, permitía controlar la textura del snack. Contenidos de humedad altos, $>42 \%$ (b.h.) producían snacks crocantes pero duros; mientras que contenidos de humedad bajos, $<26 \%$ (b.h.), producían snacks suaves y secos. Para obtener snacks de textura crocantes y suaves se debía ajustar la humedad a aproximadamente $32 \%$ (b.h.).
\end{abstract}

Palabras clave: maíz gigante, Zea mays, nixtamalización, snacks fritos, gelatinización, textura

\section{THE EFFECT OF TYPE OF PEELING AGENT, COOKING TIME AND MOISTURE CONTENT ON GIANT WHITE CORN (Zea mays) FRIED SNACK QUALITY}

\begin{abstract}
In order to improve the organoleptic properties of giant corn fried snack, the effects of chemical peeling agent type $(\mathrm{CaOH}$ or $\mathrm{NaOH})$, immersion in peeling solution, cooking time, and grain moisture content before frying, were studied.
\end{abstract}

\footnotetext{
* Departamento de Ingeniería Agroindustrial y Agronegocios, Facultad de Ingeniería, Universidad San Ignacio de Loyola, Avenida La Fontana 550, Lima 12, Perú. fcisneros@usil.edu.pe
} 
$\mathrm{NaOH}$ was found to be more effective than $\mathrm{CaOH}$ as a peeling agent of corn. Nixtamalization flavor was best developed and a lighter snack color was obtained when corn grains were immersed in the peeling solution of $\mathrm{NaOH}$ for $6 \mathrm{~h}$. A longer cooking time $(0,8,16$ min) yielded a larger grain expansion. By adjusting grain moisture content (before frying) by drying, the texture of the snack could be controlled. High moisture contents, $>42 \%$ (w.b.) produced crunchy but hard-textured snacks; whereas, low moisture contents, $<26 \%$ (w.b.) gave soft but dry-textured snacks. Therefore, in order to obtain crunchy but soft snacks, moisture content must be adjusted to approximately $32 \%$ (w.b.).

Key words: Giant corn, Zea mays, nixtamalization, fried snacks, gelatinization, texture

\section{INTRODUCCIÓN}

El maíz gigante es nativo de los Andes centrales de Sudamérica donde viene siendo cultivado desde épocas preincas, especialmente en la zona del Cusco ${ }^{1}$. Actualmente en el Perú es cultivado, principalmente, por pequeños productores para su consumo en fresco como choclo o deshidratado para su venta como mote. Este es usado como materia prima por algunos procesadores de alimentos para elaborar un snack frito nixtamalizado de grano entero de maíz gigante. Sin embargo, la textura de este tipo de snack presenta el problema de un exceso de dureza. Por lo que sería deseable que los fabricantes de este tipo de snack pudieran ajustar sus parámetros de procesamiento para reducir el nivel de dureza. La reducción de la dureza no solamente redundaría en una mejora de la calidad organoléptica del snack de maíz, sino también en reducir la posibilidad que afecte la dentadura del consumidor, especialmente el de la tercera edad.

El enfoque usado en este estudio para resolver este problema específico de calidad del snack frito de maíz gigante, es uno que utiliza el conocimiento de la ciencia de los alimentos, específicamente, del almidón y sus propiedades funcionales para formar textura en alimentos bajo diferentes condiciones de procesamiento. Una importante propiedad del almidón es la de la gelatinización, en la que el almidón interactúa con el agua bajo ciertas condiciones de calentamiento. En esta interacción, los grupos hidroxilo del almidón dejan de formar puentes de hidrógeno entre sí, y en su lugar forman puentes de hidrógeno con el agua. Dependiendo del contenido de humedad, el almidón puede formar soluciones viscosas, formar geles, o formar una matriz vítrea. Cuando, junto con el almidón, están presentes otros componentes en el alimento, entonces hay que tomar en cuenta la influencia de estos otros componentes presentes (proteínas, azúcares, grasas, fibra, etc.) en la creación de textura del alimento. Específicamente, estudiamos el efecto del tipo de agente de pelado químico $(\mathrm{NaOH}$ o $\mathrm{CaOH})$, el remojo por $6 \mathrm{~h}$ en la solución de pelado químico, tiempo de cocción $(0,8,16$ min) y contenido de humedad del grano antes de la fritura, sobre la calidad general del snack frito de maíz, especialmente su textura. 


\section{PARTE EXPERIMENTAL}

\section{Equipos}

Horno de convección marca Tagliavini, modelo Termoven de 10 bandejas (Parma, Italia). Cocina industrial de dos hornillas marca Sanson. Estufa marca JP Selecta S.A. (Barcelona, España). Equipo de Soxhlet. Texturómetro QTS-Brookfield, con accesorio barra de penetración de $4 \mathrm{~mm}$ diámetro.

\section{Reactivos}

Éter de petróleo; ácido sulfúrico, sulfato de sodio anhidro; hidróxido de sodio; ácido bórico; ácido clorhídrico. Los reactivos fueron del laboratorio Sigma-Aldrich de grado reactivo.

\section{Muestras}

Se trabajó con un lote de maíz blanco gigante grano seco de segunda (calibre: 28-32) (malla: $13,0 \mathrm{~mm}$ ) con contenido de humedad de 16,3\% (base húmeda).

\section{Proceso de elaboración de snack frito de maíz gigante}

El maíz gigante fue sometido a las operaciones de pelado químico, remojo (nixtamalización), lavado, cocción, secado, fritura, salado y envasado.

Pelado químico. Se llevó a cabo sumergiendo el maíz seco en una solución de agente alcalino a $100^{\circ} \mathrm{C}$ por un tiempo determinado, con la finalidad de aflojar y separar la cáscara (pericarpio) del grano.

Remojo o nixtamalización. El maíz se dejó sumergido en la misma solución alcalina del pelado por $6 \mathrm{~h}$, pero sin calentamiento adicional.

Lavado. Los granos de maíz fueron retirados de la solución alcalina y lavados bajo un chorro de agua potable, frotando los granos uno contra otro para poder retirar por completo los restos de cáscara del grano y eliminar el álcali residual de la superficie del grano.

Cocción. Se efectuó sumergiendo los granos en agua a $100^{\circ} \mathrm{C}$ por un tiempo determinado.

Secado. El contenido de humedad de los granos fue ajustado mediante el secado con aire caliente a $60^{\circ} \mathrm{C}$ usando un horno de convección por un tiempo determinado.

Fritura. El maíz fue frito en aceite (oleína) de palma a $180^{\circ} \mathrm{C}$, por un tiempo determinado hasta que el snack alcanzó un color ligeramente dorado. La relación maíz: aceite usada fue de 1:20.

Salado. Inmediatamente después de la fritura se espolvoreó sal en una cantidad aproximada al $1 \%$ de la masa del maíz frito, revolviendo los granos para una distribución homogénea.

Envasado. Los snacks de maíz fueron envasados en frascos de vidrio herméticos a temperatura ambiente y mantenidos en la oscuridad. 


\section{Variables de estudio}

El desarrollo de esta investigación se dividió en dos fases. La primera fase consistió en el estudio del tipo de agente químico alcalino $(\mathrm{CaOH}$ o $\mathrm{NaOH})$ en el pelado y en la nixtamalización o remojo para determinar su efecto sobre el desarrollo del sabor nixtamalizado de los snacks fritos de maíz y su efectividad en el pelado del grano.

Una vez que se determinó el tipo de agente de pelado y de nixtamalización a utilizar, se pasó a la segunda fase, que consistió en investigar los efectos del tiempo de cocción y el contenido de humedad del grano en las características de calidad de los snacks fritos de maíz, especialmente en la textura.

\section{Efecto de tipo de agente alcalino en el pelado y nixtamalización (primera fase)}

En esta primera fase se estudió el efecto del tipo de agente alcalino $(\mathrm{CaOH}$ o $\mathrm{NaOH})$ en la efectividad del pelado químico y en el desarrollo del sabor nixtamalizado y aspecto, mediante el remojo en la solución alcalina, de los snacks fritos de maíz.

Se emplearon dos condiciones de pelado: a) solución de $\mathrm{CaOH}$ al $0,8 \%(\mathrm{p} / \mathrm{v})$ en una relación maíz: solución de $1: 3$ por $45 \mathrm{~min}$ a $100^{\circ} \mathrm{C}$. b) solución de $\mathrm{NaOH} 0,5 \%(\mathrm{p} / \mathrm{v})$, relación maíz:solución de $1: 2$ por $10 \min$ a $100^{\circ} \mathrm{C}$.

Inmediatamente después del pelado, la mitad de los granos fueron sometidos a un remojo por $6 \mathrm{~h}$ en la misma solución de pelado pero sin efectuar calentamiento adicional, con el fin de desarrollar el sabor nixtamalizado en el maíz frito.

Para el desarrollo de esta fase se empleó un tiempo de cocción de 16 minutos, y no se hizo ajuste en el contenido de humedad mediante secado, ya que solo se dejó orear para la evaporación de la humedad superficial del grano.

Efecto de tiempo de cocción y contenido de humedad antes de la fritura (segunda fase) Una vez establecido el agente alcalino a utilizar y la decisión de aplicar la nixtamalización mediante remojo, entonces se pasó a la segunda fase en la que se evaluaron tres niveles de tiempo de cocción, 0,8 , y 16 min; y seis niveles de contenido de humedad (base húmeda), $17,20,30,40,45$ y $50 \%$; con lo cual se obtuvieron 18 tratamientos al aplicar un diseño completamente al azar.

El pelado, nixtamalización, lavado y oreo, se realizaron en un día. Para el oreo, los granos se esparcieron sobre unas mesas de acero inoxidable hasta al día siguiente en que se realizó la operación de cocción, y nuevamente los granos de maíz fueron sometidos a un oreo hasta al día siguiente, en que se realizó el secado. Esta operación se realizó en un día, y los granos de maíz secados se conservaron en frascos de vidrio herméticos hasta al día siguiente con el fin de que el contenido de humedad de los granos se equilibre.

La operación de fritura se realizó empleando aceite (oleína) de palma con cambio de aceite cada cuatro frituras. Con la finalidad de que los granos de maíz no flotaran en el aceite y 
poder obtener un dorado uniforme, se emplearon, además de las canastillas, unas planchas agujereadas de acero inoxidable para sumergir los granos. Los snacks fritos de maíz fueron envasados en frascos de vidrio herméticos y almacenados protegidos de la luz.

\section{Análisis químico y evaluación}

\section{Contenido de humedad}

Se hizo la determinación para las 18 muestras de maíz, tanto después del secado como en el snack frito. Se utilizó el método de estufa ${ }^{2}$ a $135^{\circ} \mathrm{C}$.

\section{Contenido de grasa cruda}

Se realizó para las 18 muestras de snacks fritos de maíz gigante, mediante el método Soxhlet ${ }^{3}$

\section{Análisis sensorial}

Se aplicó a cuatro muestras pre-seleccionadas del total de 18 muestras. La pre-selección la realizó un grupo de 13 panelistas, basándose en color y apariencia, sabor y textura. Las cuatro muestras seleccionadas fueron evaluadas mediante análisis sensorial de tipo afectivo usando escala hedónica por 50 panelistas. Los datos obtenidos del análisis sensorial fueron sometidos a análisis de varianza de una vía y la prueba de comparaciones apareadas de Tukey.

\section{Textura instrumental}

Para medir el grado de dureza del maíz frito se realizó el análisis de textura instrumental a las mismas cuatro muestras evaluadas en el análisis sensorial. Se utilizó el texturómetro QTS-Brookfield, con una barra de penetración de $4 \mathrm{~mm}$ como accesorio para comprimir y penetrar el grano hasta producir su fractura. La velocidad de penetración fue de $60 \mathrm{~mm} / \mathrm{min}$. El instrumento estuvo conectado a una computadora con un programa de recolección de datos, que automáticamente registró los datos de fuerza y distancia correspondientes.

\section{Análisis proximal}

Se realizó sobre la materia prima y el snack frito que obtuvo el mayor puntaje en el análisis sensorial. Los análisis fueron: Carbohidratos, por diferencia ${ }^{4}$; Energía total, por cálculos ${ }^{4}$; Ceniza totales ${ }^{5}$; Fibra cruda ${ }^{5}$; Grasa cruda ${ }^{5}$; Contenido de humedad ${ }^{5}$; Proteína cruda $^{5}$.

\section{RESULTADOS Y DISCUSIÓN}

\section{Efecto de tipo de agente alcalino en el pelado y nixtamalización (primera fase)}

\section{Pelado químico y nixtamalización (remojo)}

Para el pelado químico con $\mathrm{CaOH}$ se empleó una relación de maíz: solución de 1:3, relación mayor en comparación con el pelado con $\mathrm{NaOH}$, que fue de 1:2. Esta diferencia se debe a que el tiempo requerido para el pelado con $\mathrm{CaOH}$ fue de $45 \mathrm{~min}$, bajo condiciones de ebullición, con lo que el volumen inicial de la solución se reduce significativamente por evaporación. En comparación, el pelado con $\mathrm{NaOH}$ fue de sólo 10 minutos a temperatura de ebullición, y no hubo mayor cambio en el volumen de la solución. 
Una diferencia notoria entre el pelado con $\mathrm{CaOH}$ y el pelado con $\mathrm{NaOH}$, es que en el primer caso no se logra un desprendimiento completo de la cáscara del grano, por lo que para lograr un pelado completo del grano de maíz se requiere efectuar un frotamiento entre los granos. En cambio, en el pelado con $\mathrm{NaOH}$, sí se logra un desprendimiento completo de las cáscaras en la mayoría de los granos de maíz sin necesidad de frotamiento.

Los granos de maíz pelados con $\mathrm{CaOH}$, con y sin remojo, tuvieron la misma apariencia después del pelado: ausencia de cáscara, de color blanco y conservando la piloriza. Cuando los granos de maíz fueron pelados con $\mathrm{NaOH}$ pero sin remojo, aquellos se presentaron sin cáscara, de color amarillo oscuro tenue, conservando la piloriza pero oscurecida. Mientras que los granos de maíz pelados con $\mathrm{NaOH}$ y con remojo, se presentaron sin cáscara, de color amarillo claro y sin piloriza.

El grado de dureza en la textura del snack frito del maíz gigante, en esta primera etapa, fue la siguiente, siguiendo el orden de mayor dureza a menor dureza: maíz gigante pelado con $\mathrm{NaOH}$ sin remojo, maíz gigante pelado con $\mathrm{NaOH}$ con remojo, maíz gigante pelado con $\mathrm{CaOH}$ sin remojo y maíz gigante pelado con $\mathrm{CaOH}$ con remojo. Esta diferencia podría deberse a que el maíz gigante pelado con $\mathrm{CaOH}$ estuvo sometido a $100^{\circ} \mathrm{C}$ durante más tiempo (tiempo pelado, 45 min y tiempo cocción, 16 min) que con $\mathrm{NaOH}$ (tiempo pelado, 10 min y tiempo cocción, 16 min). El remojo también contribuyó a obtener una muestra más suave, considerando que comienza con una temperatura de $100^{\circ} \mathrm{C}$ para luego disminuir hasta temperatura ambiente en un lapso de seis horas.

Los rendimientos para diferentes etapas del proceso de elaboración de snacks fritos de maíz, al usar diferentes agentes de pelado con y sin remojo, se muestran en el cuadro 1. Se observa que los rendimientos aumentan después del pelado, remojo y lavado al compararlo con el grano seco inicial, lo cual es esperado debido a la absorción de agua del grano seco que ocurre principalmente durante el pelado y remojo. Sin embargo, los mayores rendimientos se observan para las muestras que fueron peladas con $\mathrm{CaOH}$, independientemente de si hubo remojo o no, en comparación con las que fueron peladas con $\mathrm{NaOH}$. Esto se debería al mayor tiempo en que los granos fueron sometidos a temperaturas de ebullición durante el pelado con $\mathrm{CaOH}$ (45 min) en comparación con $\mathrm{NaOH}$ (10 min), lo cual produjo mayor absorción de agua en los granos. El remojo en solución de $\mathrm{CaOH}$ no produjo absorción de agua adicional, probablemente debido a que el grano ya habría alcanzado su máxima absorción de agua. En cambio, cuando el grano fue pelado con $\mathrm{NaOH}$, el remojo posterior sí causó un incremento adicional del rendimiento, probablemente debido a que el corto tiempo de pelado no permitió que el grano alcanzara su máxima hidratación, entonces durante el remojo posterior continuó hidratándose.

En el cuadro 1 se observa que durante la cocción el rendimiento aumentó aún más, pero esta vez, los granos que fueron sometidos al remojo, sí mostraron un mayor rendimiento en comparación con los que no fueron sometidos a remojo, para ambos agentes de pelado. Esta diferencia se debería a que la nixtamalización (debido al remojo) de los granos de maíz, produciría cambios químicos en el almidón y en la microestructura del grano que facilitaría una mayor absorción del agua ${ }^{6}$. 
Cuadro 1. Resultados de los rendimientos de los snacks fritos por operación.

\begin{tabular}{lcclc}
\hline \multirow{2}{*}{ Operación } & \multicolumn{2}{c}{ Pelado con $\mathrm{CaOH}$} & \multicolumn{2}{c}{ Pelado con $\mathrm{NaOH}$} \\
\cline { 2 - 5 } & Sin remojo & Con remojo & Sin remojo & Con remojo \\
\hline Grano seco & $100 \%$ & $100 \%$ & $100 \%$ & $100 \%$ \\
Pelado y lavado & $153 \%$ & $153 \%$ & $130 \%$ & $140 \%$ \\
Cocción & $158 \%$ & $168 \%$ & $153 \%$ & $171 \%$ \\
Fritura & $89 \%$ & $88 \%$ & $87 \%$ & $84 \%$ \\
\hline
\end{tabular}

En el caso de la operación de la fritura el rendimiento disminuye; esto se debería a la evaporación acelerada de agua que se produce cuando los granos de maíz se ponen en contacto con el aceite caliente. Después de esta evaporación violenta del agua del grano y durante el enfriamiento posterior se genera un vacío en el interior del mismo, lo cual facilita el ingreso del aceite al interior del grano. Sin embargo, la masa de aceite absorbido no compensaría la cantidad de agua perdida ${ }^{7,8}$.

\section{Desarrollo del sabor nixtamalizado}

Para determinar el efecto del tipo de agente alcalino usado para el pelado químico y el efecto del remojo sobre la apariencia y el sabor nixtamalizado del snack de maíz frito, se evaluaron sensorialmente las cuatro muestras de snacks fritos del maíz gigante entre un grupo pequeño de panelistas (13). Se evaluó la apariencia y sabor, siendo de interés principal este último, por lo que se le pidió al grupo de panelistas que evaluaran el desarrollo del sabor nixtamalizado asociándolo con el sabor característico de un snack comercial de hojuelas de maíz nixtamalizada. En el cuadro 2 se reporta la apariencia y sabor del maíz frito de cada una de las cuatro muestras evaluadas.

Los panelistas determinaron que el pelado con $\mathrm{NaOH}$ y con remojo por $6 \mathrm{~h}$ fue el tratamiento que dio una mejor apariencia (menor oscurecimiento) y desarrolló el sabor a nixtamalización deseado. El efecto negativo sobre la apariencia se debió básicamente por el oscurecimiento del germen y la piloriza durante la fritura, que fue menor cuando se peló con $\mathrm{NaOH}$ y se aplicó remojo. Por lo tanto, para la segunda fase de este estudio se usó $\mathrm{NaOH}$ al $0,5 \%$ (p/v) por 10 min para el pelado químico, seguido de un periodo de remojo de seis horas. 
Cuadro 2. Resultados de la evaluación sensorial preliminar del snack frito de maíz gigante.

\begin{tabular}{lll}
\hline Tratamiento & Apariencia & Sabor \\
\hline $\begin{array}{l}\text { Pelado con } \mathrm{CaOH} \text { sin } \\
\text { remojo }\end{array}$ & $\begin{array}{l}\text { No muy buena, con el } \\
\text { germen completamente } \\
\text { oscurecido }\end{array}$ & Sin sabor nixtamalizado \\
$\begin{array}{l}\text { Pelado con } \mathrm{CaOH}, \text { con } \\
\text { remojo }\end{array}$ & $\begin{array}{l}\text { No muy buena, con el } \\
\text { germen oscurecido }\end{array}$ & $\begin{array}{l}\text { Ligero sabor } \\
\text { nixtamalizado }\end{array}$ \\
$\begin{array}{l}\text { Pelado con } \mathrm{NaOH}, \text { sin } \\
\text { remojo }\end{array}$ & $\begin{array}{l}\text { Regular, con el germen } \\
\text { ligeramente oscurecido }\end{array}$ & Sin sabor nixtamalizado \\
$\begin{array}{l}\text { Pelado con } \mathrm{NaOH}, \text { con } \\
\text { remojo }\end{array}$ & $\begin{array}{l}\text { Buena, los granos de color } \\
\text { dorado uniforme }\end{array}$ & $\begin{array}{l}\text { Sabor característico } \\
\text { nixtamalizado }\end{array}$ \\
\hline
\end{tabular}

\section{Efecto de tiempo de cocción y contenido de humedad antes de la fritura en la textura de snacks fritos de maíz (segunda fase)}

En esta fase del estudio se evaluaron diferentes tiempos de cocción ( 0,8 y 16 min) y de contenido de humedad del maíz antes de la fritura, mediante secado y su efecto en la aceptabilidad del snack frito.

En base a las curvas de secado, determinadas previamente, se estimó los tiempos de secado aproximados necesarios para llegar a los contenidos de humedad objetivos de 50, 45, 40, 30, 20 y $17 \%$ (b.h.). A las 18 muestras sometidas a secado se les determinó su contenido de humedad real (cuadro 3). Se puede observar que estos valores de humedad obtenidos son cercanos a los valores objetivos. También observamos que a mayor tiempo de cocción hay una tendencia a que el contenido de humedad obtenido sea ligeramente mayor para un tiempo de secado dado. Esto podría deberse a que el mayor tiempo de cocción produjo una mayor gelatinización, lo cual a su vez permitió una mayor absorción de humedad del grano, fenómeno que también pudo observarse en la primera etapa de este estudio al evaluarse los rendimientos.

Las 18 muestras de snack frito de maíz gigante obtenidas como resultado de emplear tres tiempos de cocción y seis contenidos de humedad pre-fritura, presentaron las características organolépticas descritas en el cuadro 4. Se puede observar que las 18 muestras obtenidas presentaron un color homogéneo pero diferentes apariencias debido a diferentes grados de hinchamiento de los granos fritos influenciados por los diferentes tiempos de cocción al que fueron sometidos. Los granos cocidos por un mayor tiempo (16 min), mostraron un mayor grado de hinchamiento, mientras que los granos de maíz con tiempo de cocción intermedio ( $8 \mathrm{~min}$ ) presentaron un ligero hinchamiento, y por último los granos de maíz que no fueron cocidos ( $0 \mathrm{~min})$ tuvieron una apariencia plana, es decir, un hinchamiento mínimo. Esto puede deberse a que un mayor tiempo de cocción produce un mayor grado de gelatinización, lo cual a su vez permite un mayor hinchamiento del grano ${ }^{9,10}$. Una observación importante es que los 
granos que más hincharon mostraron cavidades internas grandes, en lugar de una estructura interna uniforme formada por varias celdas pequeñas. Una posible explicación podría ser que la cocción creó unos cuantos núcleos (pequeñas cavidades) en la estructura interna del grano no frito. Estos núcleos luego sirvieron de puntos de formación de burbujas de vapor durante la fritura, y que durante el enfriamiento se convirtieron en las grandes celdas observadas.

Cuadro 3. Contenido de humedad de maíz antes de fritura, contenido de grasa cruda (b.s.), de los snacks fritos de maíz y tiempo de fritura.

\begin{tabular}{|c|c|c|c|}
\hline Muestra & $\begin{array}{c}\text { Contenido de } \\
\text { humedad (\%) (b.h.) }\end{array}$ & $\%$ grasa (b.s.) & $\begin{array}{l}\text { Tiempo de } \\
\text { fritura (s) }\end{array}$ \\
\hline $\mathrm{C} 0 \mathrm{H} 50 \%$ & $51,7 \pm 1,6$ & $15,1 \%$ & $11 \pm 1$ \\
\hline C8 H $50 \%$ & $52,1 \pm 0,6$ & $15,3 \%$ & $13 \pm 0$ \\
\hline C16 H $50 \%$ & $54.4 \pm 0,7$ & $15,4 \_\%$ & $13 \pm 0$ \\
\hline $\mathrm{C} 0 \mathrm{H} 45 \%$ & $43,9 \pm 0,4$ & $14,7 \%$ & $9 \pm 0,6$ \\
\hline C8 H $45 \%$ & $46,2 \pm 0,3$ & $15,0 \%$ & $10 \pm 0,6$ \\
\hline C16 H $45 \%$ & $46,7 \pm 1,6$ & $15,0 \%$ & $10 \pm 0$ \\
\hline $\mathrm{C} 0 \mathrm{H} 40 \%$ & $42,8 \pm 1,2$ & $14,2 \%$ & $10 \pm 0$ \\
\hline C8 H $40 \%$ & $43,8 \pm 0,5$ & $14,2 \%$ & $11 \pm 0,9$ \\
\hline C16 H $40 \%$ & $42,0 \pm 1,4$ & $14,4 \%$ & $11 \pm 0,6$ \\
\hline $\mathrm{COH} 30 \%$ & $33,6 \pm 1,7$ & $13,3 \%$ & $7 \pm 0,6$ \\
\hline C8 H $30 \%$ & $31,6 \pm 0,8$ & $13,4 \%$ & $8 \pm 0$ \\
\hline C16 H $30 \%$ & $32,6 \pm 0,5$ & $14,0 \%$ & $10 \pm 0$ \\
\hline C0 H $20 \%$ & $22,3 \pm 1,7$ & $12,3 \%$ & $6 \pm 1,3$ \\
\hline C8 H $20 \%$ & $25,9 \pm 5,2$ & $12,4 \%$ & $9 \pm 0,6$ \\
\hline C16 H $20 \%$ & $26,4 \pm 3,1$ & $13,1 \%$ & $8 \pm 1,7$ \\
\hline C0 H $17 \%$ & $18,4 \pm 0,6$ & $11,8 \%$ & $6 \pm 0$ \\
\hline C8 H $17 \%$ & $19,4 \pm 1,7$ & $12,0 \%$ & $8 \pm 0,8$ \\
\hline C16 H 17 \% & $19,3 \pm 2,1$ & $12,7 \%$ & $8 \pm 0,5$ \\
\hline
\end{tabular}

$\mathrm{C} 0=$ Tiempo de cocción 0 minutos; $\mathrm{C} 8$ = Tiempo de cocción 8 minutos; C16 = Tiempo de cocción 16 minutos; $\mathrm{H}=$ Contenido de humedad (b.h.) 
Una propiedad que tuvo una gran variación, como respuesta al contenido de humedad del grano antes de la fritura, fue la textura, ya que las muestras más húmedas (40, 45, y $50 \%$ ) fueron muy duras y crocantes, mientras que las muestras menos húmedas (17 y $20 \%$ ) fueron secas y suaves. Una posible explicación es que los granos con alto contenido de humedad al ser calentados rápidamente durante la fritura produjeron una evaporación de agua masiva, creando burbujas que crecieron rápidamente hasta el punto de producir una coalescencia extensiva de burbujas, resultando en la presencia de pequeña cantidad de grandes celdas con paredes gruesas en la estructura interna del producto frito final. Por otra parte, los granos con bajo contenido de humedad produjeron una evaporación más limitada de vapor de agua, y por tanto, burbujas de vapor pequeñas que no causaron mayor coalescencia de burbujas, lo cual condujo a la formación de numerosas celdas pequeñas de paredes delgadas en la estructura interna del producto frito final. Las paredes gruesas de las celdas grandes serían las responsables de la textura dura del grano frito, mientras que las paredes delgadas de las celdas pequeñas serían responsables de la textura suave ${ }^{11}$.

Cabe indicar que el tiempo de cocción tuvo una influencia menor en la textura del maíz frito comparado con el contenido de humedad del grano antes de la fritura. 
Cuadro 4. Características organolépticas de diferentes muestras del snack frito del maíz gigante, para diferentes tiempos de cocción y humedades antes de la fritura.

\begin{tabular}{|c|c|c|c|}
\hline Muestra & Color y apariencia & Sabor & Textura \\
\hline $\mathrm{C} 0 \mathrm{H} 50 \%$ & $\begin{array}{l}\text { Color dorado uniforme, el grano se } \\
\text { presenta de forma plana circular. }\end{array}$ & $\begin{array}{l}\text { Característico a } \\
\text { nixtamalizado. }\end{array}$ & $\begin{array}{l}\text { Muy duro y crocante. } \\
\text { Inaceptable. }\end{array}$ \\
\hline C8 H $50 \%$ & $\begin{array}{l}\text { Color dorado uniforme, el grano se } \\
\text { presenta ligeramente hinchado. }\end{array}$ & $\begin{array}{l}\text { Característico a } \\
\text { nixtamalizado. }\end{array}$ & $\begin{array}{l}\text { Muy duro y crocante } \\
\text { Inaceptable. }\end{array}$ \\
\hline C16 H 50\% & $\begin{array}{l}\text { Color dorado uniforme, el grano se } \\
\text { presenta completamente hinchado. }\end{array}$ & $\begin{array}{l}\text { Característico a } \\
\text { nixtamalizado. }\end{array}$ & $\begin{array}{l}\text { Muy duro y crocante } \\
\text { Inaceptable. }\end{array}$ \\
\hline $\mathrm{C} 0 \mathrm{H} 45 \%$ & $\begin{array}{l}\text { Color dorado uniforme, el grano se } \\
\text { presenta de forma plana circular. }\end{array}$ & $\begin{array}{l}\text { Característico a } \\
\text { nixtamalizado. }\end{array}$ & $\begin{array}{l}\text { Muy duro y crocante } \\
\text { Inaceptable. }\end{array}$ \\
\hline $\mathrm{C} 8 \mathrm{H} 45 \%$ & $\begin{array}{l}\text { Color dorado uniforme, el grano se } \\
\text { presenta ligeramente hinchado. }\end{array}$ & $\begin{array}{l}\text { Característico a } \\
\text { nixtamalizado. }\end{array}$ & $\begin{array}{l}\text { Muy duro y crocante } \\
\text { Inaceptable. }\end{array}$ \\
\hline C16 H 45\% & $\begin{array}{l}\text { Color dorado uniforme, el grano se } \\
\text { presenta completamente hinchado. }\end{array}$ & $\begin{array}{l}\text { Característico a } \\
\text { nixtamalizado. }\end{array}$ & $\begin{array}{l}\text { Duro y crocante. } \\
\text { Aceptable. }\end{array}$ \\
\hline $\mathrm{C} 0 \mathrm{H} 40 \%$ & $\begin{array}{l}\text { Color dorado uniforme, el grano se } \\
\text { presenta de forma plana circular. }\end{array}$ & $\begin{array}{l}\text { Característico a } \\
\text { nixtamalizado. }\end{array}$ & $\begin{array}{l}\text { Duro y crocante. } \\
\text { Aceptable. }\end{array}$ \\
\hline $\mathrm{C} 8 \mathrm{H} 40 \%$ & $\begin{array}{l}\text { Color dorado uniforme, el grano se } \\
\text { presenta ligeramente hinchado. }\end{array}$ & $\begin{array}{l}\text { Característico a } \\
\text { nixtamalizado. }\end{array}$ & $\begin{array}{l}\text { Duro y crocante. } \\
\text { Aceptable. }\end{array}$ \\
\hline $\mathrm{C} 16 \mathrm{H} 40 \%$ & $\begin{array}{l}\text { Color dorado uniforme, el grano se } \\
\text { presenta completamente hinchado. }\end{array}$ & $\begin{array}{l}\text { Característico a } \\
\text { nixtamalizado. }\end{array}$ & $\begin{array}{l}\text { Duro y crocante. } \\
\text { Aceptable. }\end{array}$ \\
\hline $\mathrm{CO} \mathrm{H} 30 \%$ & $\begin{array}{l}\text { Color dorado uniforme, el grano se } \\
\text { presenta de forma plana circular. }\end{array}$ & $\begin{array}{l}\text { Característico a } \\
\text { nixtamalizado. }\end{array}$ & $\begin{array}{l}\text { Un poco duro y } \\
\text { crocante. Aceptable. }\end{array}$ \\
\hline C8 H $30 \%$ & $\begin{array}{l}\text { Color dorado uniforme, el grano se } \\
\text { presenta ligeramente hinchado. }\end{array}$ & $\begin{array}{l}\text { Característico a } \\
\text { nixtamalizado. }\end{array}$ & $\begin{array}{l}\text { Un poco duro y } \\
\text { crocante. Aceptable. }\end{array}$ \\
\hline C16 H $30 \%$ & $\begin{array}{l}\text { Color dorado uniforme, el grano se } \\
\text { presenta completamente hinchado. }\end{array}$ & $\begin{array}{l}\text { Característico a } \\
\text { nixtamalizado. }\end{array}$ & $\begin{array}{l}\text { Un poco duro y } \\
\text { crocante. Aceptable. }\end{array}$ \\
\hline $\mathrm{C} 0 \mathrm{H} 20 \%$ & $\begin{array}{l}\text { Color dorado uniforme, el grano se } \\
\text { presenta de forma plana circular. }\end{array}$ & $\begin{array}{l}\text { Característico a } \\
\text { nixtamalizado. }\end{array}$ & $\begin{array}{l}\text { Seco y un poco } \\
\text { suave. Aceptable. }\end{array}$ \\
\hline C8 H $20 \%$ & $\begin{array}{l}\text { Color dorado uniforme, el grano se } \\
\text { presenta ligeramente hinchado. }\end{array}$ & $\begin{array}{l}\text { Característico a } \\
\text { nixtamalizado. }\end{array}$ & $\begin{array}{l}\text { Seco y un poco } \\
\text { suave. Aceptable. }\end{array}$ \\
\hline C16 H $20 \%$ & $\begin{array}{l}\text { Color dorado uniforme, el grano se } \\
\text { presenta completamente hinchado. }\end{array}$ & $\begin{array}{l}\text { Característico a } \\
\text { nixtamalizado. }\end{array}$ & $\begin{array}{l}\text { Seco y un poco } \\
\text { suave. Aceptable. }\end{array}$ \\
\hline $\mathrm{C} 0 \mathrm{H} 17 \%$ & $\begin{array}{l}\text { Color dorado uniforme, el grano se } \\
\text { presenta de forma plana circular. }\end{array}$ & $\begin{array}{l}\text { Característico a } \\
\text { nixtamalizado. }\end{array}$ & $\begin{array}{l}\text { Seco y suave. } \\
\text { Aceptable. }\end{array}$ \\
\hline C 8 H $17 \%$ & $\begin{array}{l}\text { Color dorado uniforme, el grano se } \\
\text { presenta ligeramente hinchado. }\end{array}$ & $\begin{array}{l}\text { Característico a } \\
\text { nixtamalizado. }\end{array}$ & $\begin{array}{l}\text { Seco y suave. } \\
\text { Aceptable. }\end{array}$ \\
\hline C16 H 17\% & $\begin{array}{l}\text { Color dorado uniforme, el grano se } \\
\text { presenta completamente hinchado. }\end{array}$ & $\begin{array}{l}\text { Característico a } \\
\text { nixtamalizado. }\end{array}$ & $\begin{array}{l}\text { Seco y suave. } \\
\text { Aceptable. }\end{array}$ \\
\hline
\end{tabular}




\section{Análisis del snack frito de maíz gigante}

\section{Contenido de grasa}

En el cuadro 3 se muestran los contenidos de grasa después de la fritura y el contenido de humedad antes de la fritura de las 18 muestras sometidas a distintos tiempos de cocción y de secado. Se puede observar que hay una buena correlación entre el contenido de grasa del grano frito y su contenido de humedad previo a la fritura (figura 1), tal como se ha establecido plenamente en estudios anteriores para otras frituras ${ }^{12}$. Es decir, manipulando los parámetros de contenido de humedad del maíz antes de la fritura y el tiempo de cocción, podemos controlar el contenido de grasa final del snack frito de maíz gigante dentro de cierto rango.

El agua contenida en el maíz, al entrar en contacto con el aceite caliente, sale en forma de vapor, permitiendo el ingreso del aceite al espacio dejado por el agua al interior del producto $^{7,8}$. Por ello, las muestras fritas con mayor contenido de aceite correspondieron a aquellas muestras que tuvieron un mayor contenido de humedad antes de la fritura.

El tiempo de fritura parece estuvo influenciado por el contenido de humedad del grano de maíz antes de la fritura, a mayor contenido de humedad, mayor fue el tiempo de fritura requerido (cuadro 3 ).

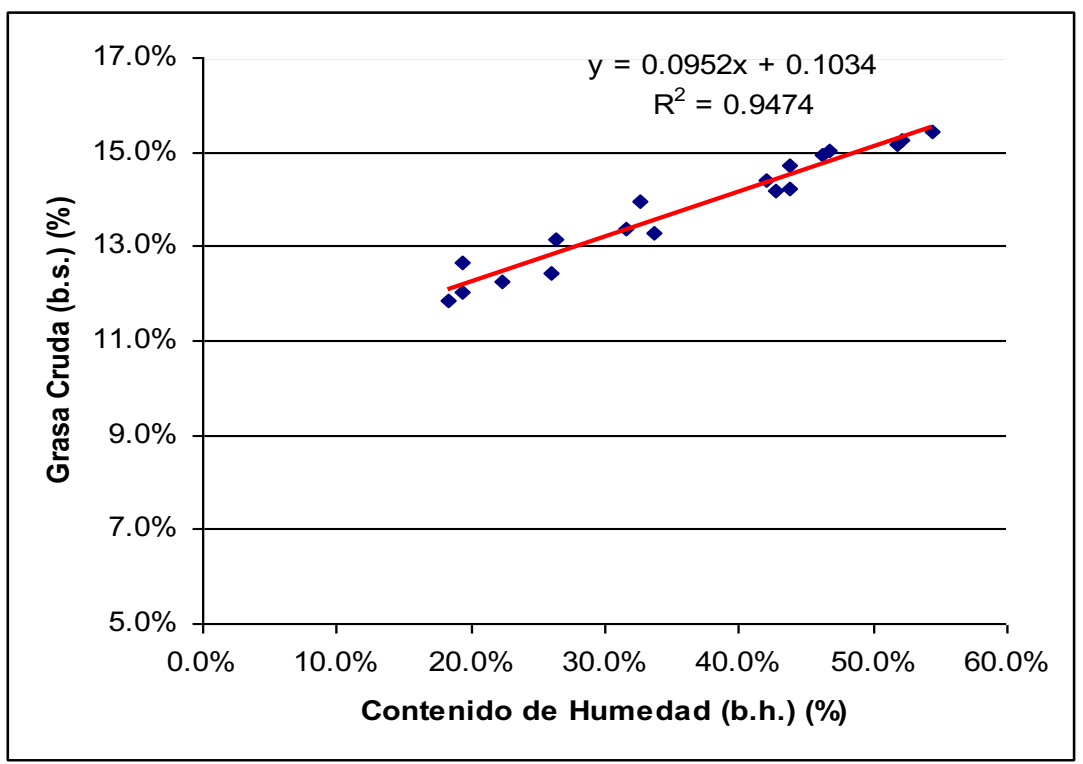

Figura 1. Correlación entre grasa cruda \% (b.s.) del snack frito de maíz gigante y el contenido de humedad \% (b.h.) del maíz gigante antes de la fritura. 
Durante la fritura, parte del agua del alimento se transforma en vapor y se elimina mediante burbujeo a través del aceite, que disminuye gradualmente a medida que el alimento se cocina en el aceite. Cantidades excesivas de humedad en la superficie del alimento produce un burbujeo violento y también facilita la liberación de los ácidos grasos libres que contribuyen a un deterioro temprano del aceite ${ }^{13}$. Por esta razón, se frieron primero los granos de maíz con menor contenido de humedad y al final los de mayor humedad, de tal manera que la hidrólisis de los triglicéridos del aceite no ocurriera tempranamente durante la fritura.

\section{Análisis sensorial de snacks fritos de maíz}

El análisis sensorial se aplicó a 4 de las 18 muestras. La selección de las cuatro muestras se basó en la obtención de muestras con diferentes contenidos de humedad y con un tiempo de cocción de 16 min. La evaluación sensorial de las muestras seleccionadas fue llevada a cabo por 50 panelistas. En el cuadro 5 se puede observar el puntaje promedio de cada atributo (color y apariencia, sabor y textura), así como el puntaje total promedio de la suma de los tres atributos para cada muestra.

Cuadro 5. Puntaje total del promedio de las muestras de snack frito de maíz gigante.

\begin{tabular}{|c|c|c|c|c|}
\hline \multirow{2}{*}{ Atributos } & \multicolumn{4}{|c|}{ Muestras } \\
\hline & C $16 \mathrm{H} 45 \%$ & C $16 \mathrm{H} 40 \%$ & С 8 H $30 \%$ & C 16 H $20 \%$ \\
\hline $\begin{array}{l}\text { Color y } \\
\text { apariencia }\end{array}$ & $5,64^{\mathrm{a}}$ & $6,04^{\mathrm{a}}$ & $5,56^{\mathrm{a}}$ & $6,10^{\mathrm{a}}$ \\
\hline Sabor & $6,36^{\mathrm{a}}$ & $5,84^{\mathrm{a}}$ & $6,10^{\mathrm{a}}$ & $5,82^{\mathrm{a}}$ \\
\hline Textura & $5,52^{\mathrm{a}}$ & $5,68^{\mathrm{ab}}$ & $6,64^{b}$ & $6,36^{\mathrm{ab}}$ \\
\hline Puntaje total & $17,56^{\mathrm{a}}$ & $17,56^{\mathrm{a}}$ & $18,30^{\mathrm{a}}$ & $18,28^{a}$ \\
\hline \multicolumn{5}{|c|}{$\begin{array}{l}\text { C } 16 \text { H } 45 \% \text { = Tiempo de cocción } 16 \text { minutos y contenido de humedad de } 45 \% \text {; C } 16 \text { H } 40 \%=\text { Tiempo } \\
\text { de cocción } 16 \text { minutos y contenido de humedad de } 40 \% \text {; C } 8 \text { H } 30 \%=\text { Tiempo de cocción } 8 \text { minutos y } \\
\text { contenido de humedad de } 30 \% \text {; C } 16 \text { H } 20 \%=\text { Tiempo de cocción } 16 \text { minutos y contenido de humedad } \\
\text { de } 20 \% \text {. }\end{array}$} \\
\hline
\end{tabular}

Los puntajes de color y apariencia, así como sabor, de las cuatro muestras (cuadro 5), no mostraron diferencias significativas ( $\mathrm{p}>0,05)$. En cambio las muestras $\mathrm{C} 8 \mathrm{H} 30 \%$ y C16H20 \%, sí mostraron diferencias significativas con respecto a $\mathrm{C} 16 \mathrm{H} 45 \%$ y $\mathrm{C} 16 \mathrm{H} 40 \%$, en cuanto a textura $(p>0,05)$. En este caso se puede ver el efecto del contenido de la humedad antes de la fritura sobre la textura del maíz frito. Las dos primeras muestras, con un contenido de humedad nominal más bajo (20 y $30 \%$ ), tuvieron un puntaje significativamente más alto que las dos últimas muestras, con contenido de humedad nominal más alto (40 y $45 \%$ ). 
De acuerdo a los comentarios de los panelistas, las muestras de snack frito de maíz C16H40 \% y $\mathrm{C} 16 \mathrm{H} 45 \%$ tuvieron una textura algo dura, mientras que la muestra $\mathrm{C} 8 \mathrm{H} 30 \%$ tuvo una textura crocante y suave; en tanto, la muestra de $\mathrm{C} 16 \mathrm{H} 20 \%$ exhibió una textura no tan crocante pero suave. Cabe indicar que los contenidos de humedad reales de las muestras $\mathrm{C} 16 \mathrm{H} 45, \mathrm{C} 16 \mathrm{H} 40, \mathrm{C} 8 \mathrm{H} 30$ y C16H20 fueron 47, 42, 32 y $26 \%$, respectivamente, según se puede apreciar en el cuadro 3.

En resumen, entre los atributos evaluados sensorialmente de color y apariencia, sabor y textura del snack frito de maíz gigante, sólo los puntajes correspondientes a textura, mostraron diferencias significativas entre las muestras evaluadas, siendo las muestras C8H30 \% (tiempo de cocción 8 min y contenido de humedad nominal $30 \%$ ) y C16H20 \% (tiempo de cocción 16 min y contenido de humedad nominal $20 \%$ ) las que obtuvieron los puntajes más altos. Cabe resaltar que estas fueron las muestras con contenido de humedad antes de la fritura reales en el rango 26 - $32 \%$; valores de humedad mayores dieron como resultados snacks fritos de mayor dureza.

\section{Textura instrumental}

Según los resultados obtenidos en el análisis de textura instrumental, la muestra que presentó mayor fuerza del pico fue $\mathrm{C} 16 \mathrm{H} 45$ con $58,8 \mathrm{~N} / \mathrm{mm}$, seguido de $\mathrm{C} 16 \mathrm{H} 40 \%$ con 53,5 N/ $\mathrm{mm}, \mathrm{C} 8 \mathrm{H} 30 \%$ con $42,9 \mathrm{~N} / \mathrm{mm}$ y por último $\mathrm{C} 16 \mathrm{H} 20 \%$ con $38,0 \mathrm{~N} / \mathrm{mm}$. Estos resultados establecen que a mayor contenido de humedad antes de la fritura, mayor es la fuerza del pico para el snack frito de maíz gigante. Además, estos resultados confirman los comentarios de los panelistas de la evaluación sensorial en cuanto a la dureza de los snacks.

\section{CONCLUSIONES}

En conclusión, se determinó que $\mathrm{NaOH}$ es más eficiente que $\mathrm{CaOH}$ para el pelado químico del maíz, y cuando se dejaban remojando los granos por $6 \mathrm{~h}$ en la solución de pelado de $\mathrm{NaOH}$, se desarrollaba mejor el sabor nixtamalizado, además de obtenerse un color más claro del snack de maíz. Un mayor tiempo de cocción del maíz $(0,8,16$ min) produjo una mayor expansión del snack, y el ajuste de la humedad mediante el secado, previo a la fritura, permitió controlar la textura del snack. Contenidos de humedad del maíz (antes de la fritura) altos, $42-47 \%$ (b.h.) producían snacks crocantes pero duros; mientras que contenidos de humedad bajos, menores a $26 \%$ (b.h.), producían snacks suaves y secos. Para obtener snacks de textura crocantes y suaves, se debía ajustar el contenido de humedad a aprox. 32 \% (b.h.). 


\section{REFERENCIAS BIBLIOGRÁFICAS}

1. Productores y Mercados del Agro de la Sierra del Perú . Estudio Histórico Cultural sobre el Maíz Blanco Gigante Cusco. [Internet]. Servicio de Gestión del Conocimiento para Latinoamérica y el Caribe; s/f. [Citado el 25 Nov 2017]. Disponible en: http://www. asocam.org/biblioteca/P0131_completo.pdf.

2. American Association of Cereal Chemists (AACC). Approved Methods of the AACC, 9th ed. St. Paul MN: American Association of Cereal Chemists; 1995

3. Association of Official Analytical Chemists (AOAC). Official Methods of Analysis, 15th ed. Washington: Association of Official Analytical Chemist; 1990.

4. Collazos C. La composición de alimentos de mayor consumo en el Perú. 6ta ed. Lima: Ministerio de Salud, Instituto Nacional de Nutrición; 1993.

5. FAO. Food Analysis: General techniques, additives, contaminants and composition. FAO Food and Nutrition Paper, Vol. 14/7. Roma (Italia); 1986.

6. Gómez MH, Lee JK, McDonough CM, Waniska RD, Rooney LW. Corn starch changes during tortilla and tortilla chip processing. Cereal Chem. 1992; 69(3): 275-279.

7. Bouchon PB, Aguilera JM, Pyle DL. Structure Oil-Absorption Relationships During Deep-Fat Frying. J Food Sci. 2003; 68(9): 2711-2716.

8. Moreno MC, Bouchon PB. A Different Perspective to Study the Effect of Freeze, Air, and Osmotic Drying on Oil Absorption during Potato Frying. J Food Sci. 2008; 73(3): E122-E128.

9. Case SE, Hamann DD, Schwartz SJ. Effect of starch gelatinization on physical properties of extruded wheat- and corn-based products. Cereal Chem. 1992; 69(4): 401-404.

10. Mason WR. Starch use in foods. En Starch: Chemistry and Technology, 3ra Ed. BeMiller J, Whistler R. (Eds.). Burlington, MA: Elsevier; 2009.

11. Gao X, Tan J, Shatadal P, Heymann H. Evaluating expanded-food sensory properties by image analysis. J Texture Studies. 1999; 30(3): 291-304.

12. Ziaiifar AM, Achir N, Courtois F, Trezzani I, Trystam G. Review of mechanisms, conditions, and factors involved in the oil uptake phenomenon during the deep-fat frying process. Int J Food Sci \& Technol. 2008; 43(8): 1410-1423.

13. Lawson H. Fritura por Inmersión. En Aceites y Grasas Alimentarios. Tecnología, Utilización y Nutrición. Zaragoza: Ed. Acribia; 1996. 